\title{
Aus dem Vorwort zur ersten Auflage
}

Das Allgemeine Verwaltungsrecht mit seinen Rechtsinstituten, seinen Grundsätzen und seiner inneren Systematik muß sich an dem Fortgang der Staatsaufgaben und an der Entwicklung der Rechtsformen des Verwaltungshandelns orientieren. Autoren und Herausgeber haben sich das Ziel gesetzt, die damit gestellten Anforderungen zu erreichen. Das Buch ist zuerst auf die Bedürfnisse der Studenten zugeschnitten. Ihnen will es allerdings mehr geben als eine Einführung oder ein Kurzlehrbuch. Auf der anderen Seite bringt es die Absicht, ein Hilfsmittel für Studium und Prüfung zur Verfügung zu stellen, mit sich, daß nach Stoffverarbeitung und Darstellung nicht die Ansprüche eines großen Lehrbuchs oder Handbuchs angestrebt werden. Autoren und Herausgeber haben freilich auch das Ziel verfolgt, durch die selbständige Behandlung des umfangreichen Materials und durch die Auseinandersetzung mit Literatur und Rechtsprechung zur wissenschaftlichen Durchdringung des Allgemeinen Verwaltungsrechts beizutragen und dem Interesse der Praxis an den dogmatischen Grundlagen und Zusammenhängen des Verwaltungsrechts entgegenzukommen.

Das Werk ist eine Gemeinschaftsarbeit. Autoren und Herausgebern war von Anbeginn klar, daß die Gesamtdarstellung des Allgemeinen Verwaltungsrechts durch mehrere Autoren ein Wagnis ist. Diese Überzeugung hat sich im Verlauf der Entstehung des Werkes bestätigt und noch verstärkt. Sie hoffen aber, daß es - bei aller Unterschiedlichkeit der acht Autoren in einzelnen Standpunkten - gelungen ist, ein Werk zustande zu bringen, das durch die Verbindung systematischen Vorgehens mit eingearbeiteten Fällen und Beispielen sowohl eine Veranschaulichung der Fragestellungen und Probleme des Allgemeinen Verwaltungsrechts als auch eine wissenschaftliche Fundierung dieses Rechtsgebiets fördern kann. 